\title{
Correction: What does expert opinion in guidelines mean? a meta-epidemiological study
}

Ponce OJ, Alvarez-Villalobos N, Shah R, et al. What does expert opinion in guidelines mean? a meta-epidemiological study. BMJ Evidence-Based Medicine 2017;22:164-169. doi: 10.1136/ ebmed-2017-110798

In the Results section, the sentence 'Having a methodologist as a part of a guideline team was associated with increased rate of EO recommendations per guideline ( $\mathrm{p}=0.03)$, with using 'lack of evidence' as a rationale for the EO recommendations...' should read 'Having a methodologist as a part of a guideline team was associated with decreased rate of EO recommendations per guideline $(\mathrm{P}=0.03)$, with increased rate of using 'lack of evidence' as a rationale for the EO recommendations.... In the Discussion section, the sentence 'This study has also demonstrated that having a methodologist as a part of a guideline team was associated with increased rate of EO recommendations per guideline' should read 'This study has also demonstrated that having a methodologist as a part of a guideline team was associated with decreased rate of EO recommendations per guideline'. In addition, the sentence 'This may signify that rating of the quality of evidence was either controversial, sparse or of low quality, which required increased use of experts' input', should be removed.

( $)$ Article author(s) (or their employer(s) unless otherwise stated in the text of the article) 2018. All rights reserved. No commercial use is permitted unless otherwise expressly granted.

BMJ Evidence-Based Medicine 2018;23:78. doi:10.1136/ebmed-2017-110798corr1

A) Check for updates 